\title{
Archéopages
}

Archéopages

Archéologie et société

42 | 04-07/2015

Construire en terre crue

\section{Briques crues et « terre massive » dans le nord de la France}

Deux celliers gallo-romains de la Plaine de France

Mudbricks and "solid earth" constructions in northern France. Two Gallo-

Roman cellars in Île-de-France

Ladrillos de barro y «tierra maciza» en el norte de Francia. Dos bodegas

galorromanas de la Llanura de Francia

\section{Gaëlle Bruley-Chabot}

\section{OpenEdition \\ Journals}

Édition électronique

URL : https://journals.openedition.org/archeopages/1223

DOI : 10.4000/archeopages. 1223

ISSN : 2269-9872

Éditeur

INRAP - Institut national de recherches archéologiques préventives

Édition imprimée

Date de publication : 1 mars 2016

Pagination : 70-77

ISSN : 1622-8545

Référence électronique

Gaëlle Bruley-Chabot, «Briques crues et « terre massive » dans le nord de la France », Archéopages [En ligne], 42 | 04-07/2015, mis en ligne le 01 juillet 2017, consulté le 02 juin 2021. URL : http://

journals.openedition.org/archeopages/1223 ; DOI : https://doi.org/10.4000/archeopages.1223 


\section{Briques crues et « terre massive » dans le nord de la France Deux celliers gallo-romains de la Plaine de France}

Gaëlle Bruley-Chabot Inrap, UMR 7041, «ArScAn»

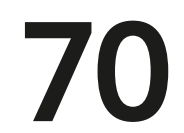

1. La terminologie employée ici se réfère à l'article d'Aurenche et coll., 2011.

2. Fouille menée en 2008 sous la direction de Gaëlle Bruley-Chabot.
Les constructions de terre observées en Îlede-France, de la Protohistoire jusqu'à l'époque moderne, sont dans la majorité des cas interprétées comme étant constituées de parois de torchis - terre à dégraissant végétal dominant - apposé sur un clayonnage. La présence conjointe de trous de poteau et la découverte de nombreux fragments de torchis brûlés, en position secondaire, sont la base de notre argumentaire pour ce type de mise en œuvre. Cependant, tous les éléments de terre rubéfiée ne témoignent pas d'empreintes de clayonnages. Les dégraissants montrent une grande variabilité. Les fragments retrouvés peuvent donc provenir de la destruction de parois construites, d'objets manufacturés en terre voire de sédiment rubéfié en place (Duvernay, 2003). Deux celliers gallo-romains, mis au jour à Épiaislès-Louvres « la Fosse » et Bonneuil-en-France «la Fontaine Plamont» (Val-d'Oise), illustrent, une fois n'est pas coutume, des exemples de mises en œuvre en terre crue retrouvés en position primaire. Ces deux sites sont implantés sur les terrains limoneux de la Plaine de France, au nord de Paris. Le premier est daté du II ${ }^{\mathrm{e}}$-début IV siècle et le second du I ${ }^{\text {er }}$ - fin $\mathrm{IV}^{\mathrm{e}}$ siècle. Le caractère excavé de ces structures, les préservant de l'érosion, est sans doute à l'origine de cette bonne conservation. Ces exemples permettent de s'interroger sur une utilisation de la terre crue sous une autre forme que le torchis.

Une cloison en brique crue à Épiais-lès-Louvres L'occupation d'Épiais-lès-Louvres «la Fosse » s'inscrit dans un enclos de $5500 \mathrm{~m}^{2}$ et se caractérise par son activité de forge ${ }^{2}$ (BruleyChabot, 2013). Établi à proximité d'une voie, l'atelier est associé à un grand bâtiment sur poteaux et à plusieurs caves. Ces installations permettent vraisemblablement d'accueillir les clients de la forge ainsi que leur monture. Chaque cave témoigne de creusements et d'aménagements particuliers. C'est au sein de l'une d'elles qu'une cloison de terre crue a été observée.

La cave 4013 a été creusée dans un limon jaune orangé. De forme carrée, la structure est conservée sur 1,40 $\mathrm{m}$ de profondeur et présente un fond plat et des bords droits. L'analyse stratigraphique a mis en évidence deux états d'utilisation [ill. 1]. Durant la première phase, le fond est aménagé de treize cupules plus ou moins circulaires pouvant servir à caler des vases ou des paniers. On accède à cette cave au moyen d'un escalier composé de quatre marches façonnées dans le substrat limoneux. Un mur de pierre a été construit contre la paroi adjacente. Large de $30 \mathrm{~cm}$, il est constitué de moellons calcaires et de silex très grossièrement équarris (sauf pour les pierres de l'angle nord), de moyennes dimensions, liés par un limon brun. Le mur est conservé sur sept assises, ce qui correspond à une hauteur de $70 \mathrm{~cm}$ [ill. 2].

La seconde phase succède à l'effondrement du mur en pierre qui a été laissé en place. Le niveau du sol a été égalisé à l'aide d'une couche de limon jaune orangé équivalent au limon encaissant. Le sol a ainsi été rehaussé d'une hauteur équivalente à la première marche. Une nouvelle partition est alors opérée, divisant l'espace en deux loges. La cloison, qui est perpendiculaire à l'escalier, a été construite à l'aide de briques crues faites d'un limon argileux gris vert, sans dégraissant apparent [ill. 3 et 4]. Les briques mises en évidence, épaisses de $10 \mathrm{~cm}$ environ, sont disposées en cinq assises. Leurs largeurs semblent correspondre à celle du muret, soit $38,40 \mathrm{~cm}$. La cloison devait être plus haute car de nombreux fragments ont été identifiés dans le comblement des loges. Les éléments les mieux 


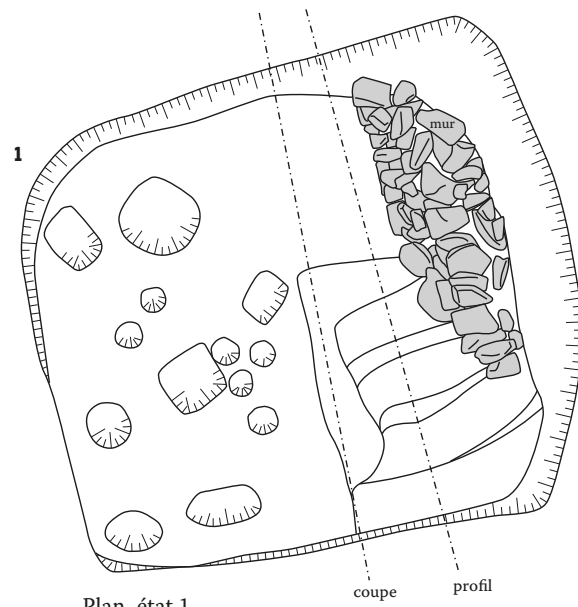

Plan état 1

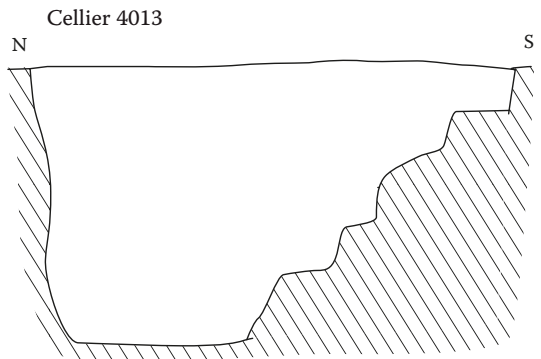

profil de l'escalier creusé dans le limon orangé encaissant

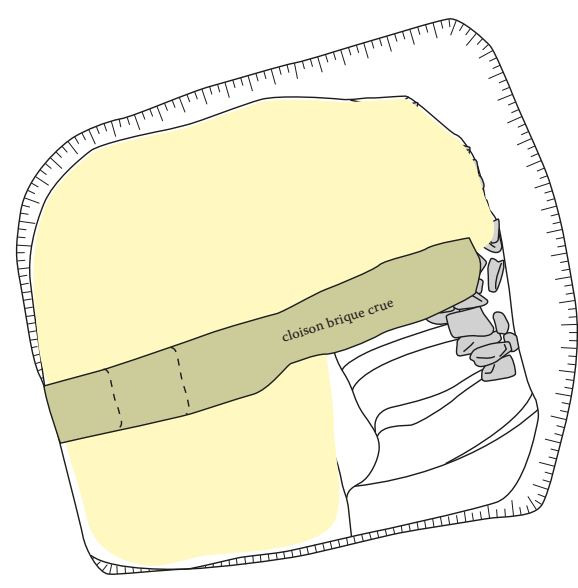

Plan état 2
Limon orangé plus ou moins hétérogène

Pierres calcaires

Brique crue, argile grise verte

Brique crue, limon orangé

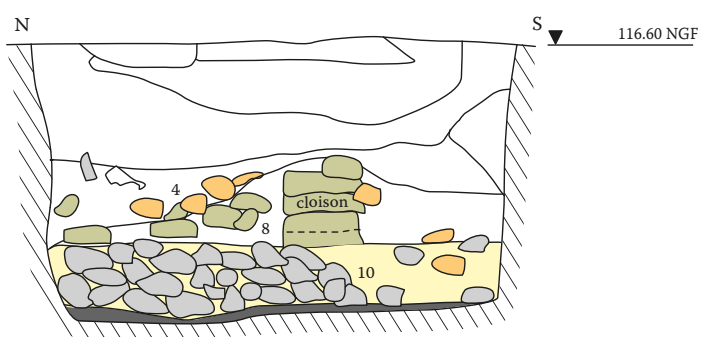

coupe stratigraphique du cellier

1. Le cellier $4013 \mathrm{~d}$ d'Épiaislès-Louvres (Val-d'Oise)

s'étend sur 2,70 $\mathrm{m}$ de long et $2,40 \mathrm{~m}$ de large.

2. Dans son premier état,

caractérisé par un mur en pierre et des cupules, le cellier d'Épiais-lès-Louvres offrait une surface

utilisable de $3,40 \mathrm{~m}^{2}$ : on constate que les

cupules se concentrent

sur une seule moitié, hors

de la zone de circulation

de l'accès.
3. Le deuxième état de la 4. La forte homogénéité cave d’Épiais-lès-Louvres du mélange rend difficile est caractérisé par une la lecture des joints entre

cloison de briques crues les briques. À Épiais-lèsconservée sur toute la longueur du cellier, soit $2,28 \mathrm{~m}$, sur une largeur de 0,38 m et une hauteur de $46 \mathrm{~cm}$, ce qui a permis de calculer le volume de terre nécessaire à sa confection.
Louvres, la coupe

stratigraphique a été

déterminante dans

la reconnaissance des

modules, les joints entre

les assises étant plus épais

et plus foncés.
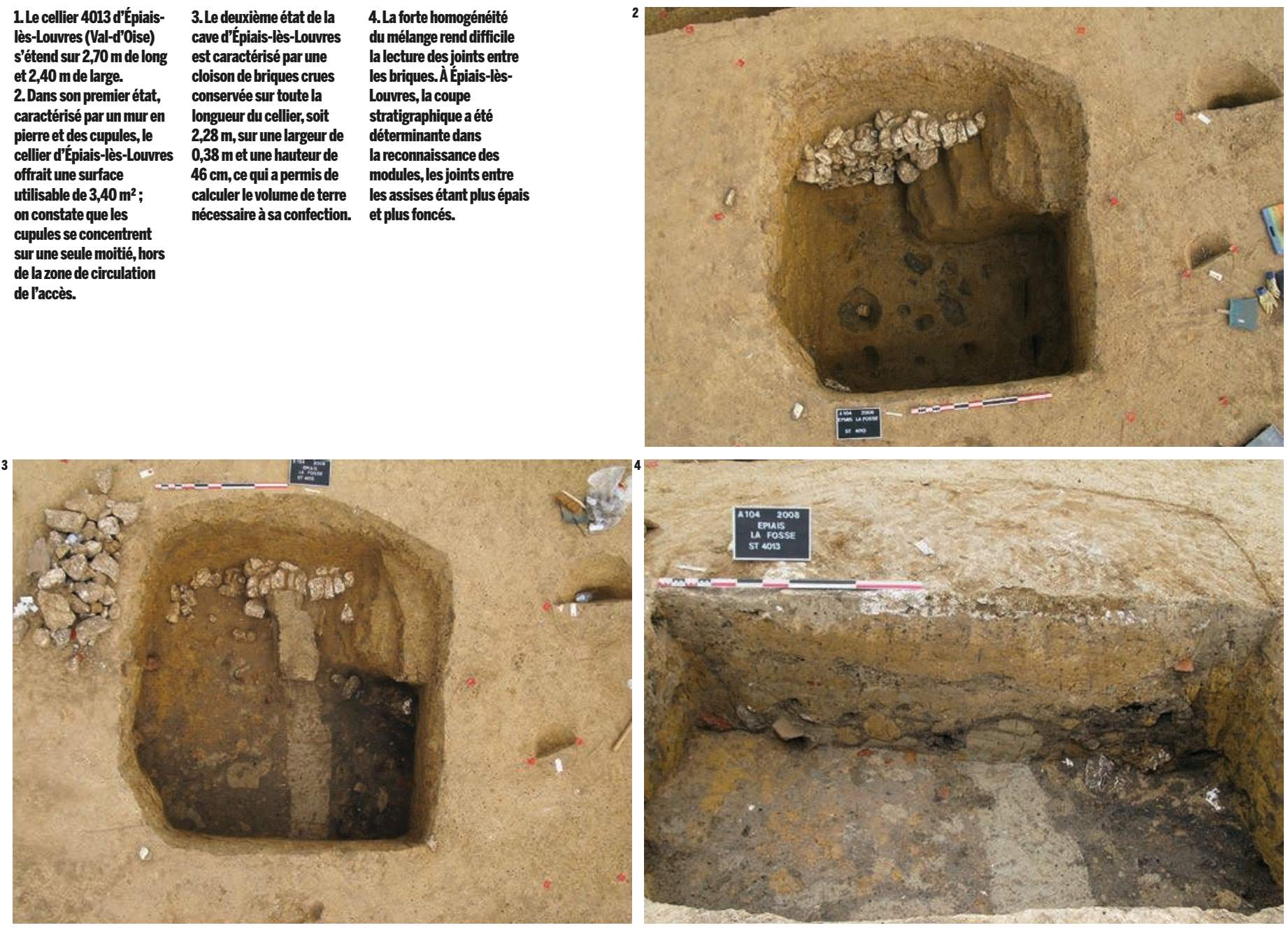


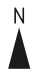

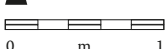

Planches bois carbonisées

Limon brun hétérogène

Pierres calcaires

Brique crue, limon orangé

$\mathbf{N}$
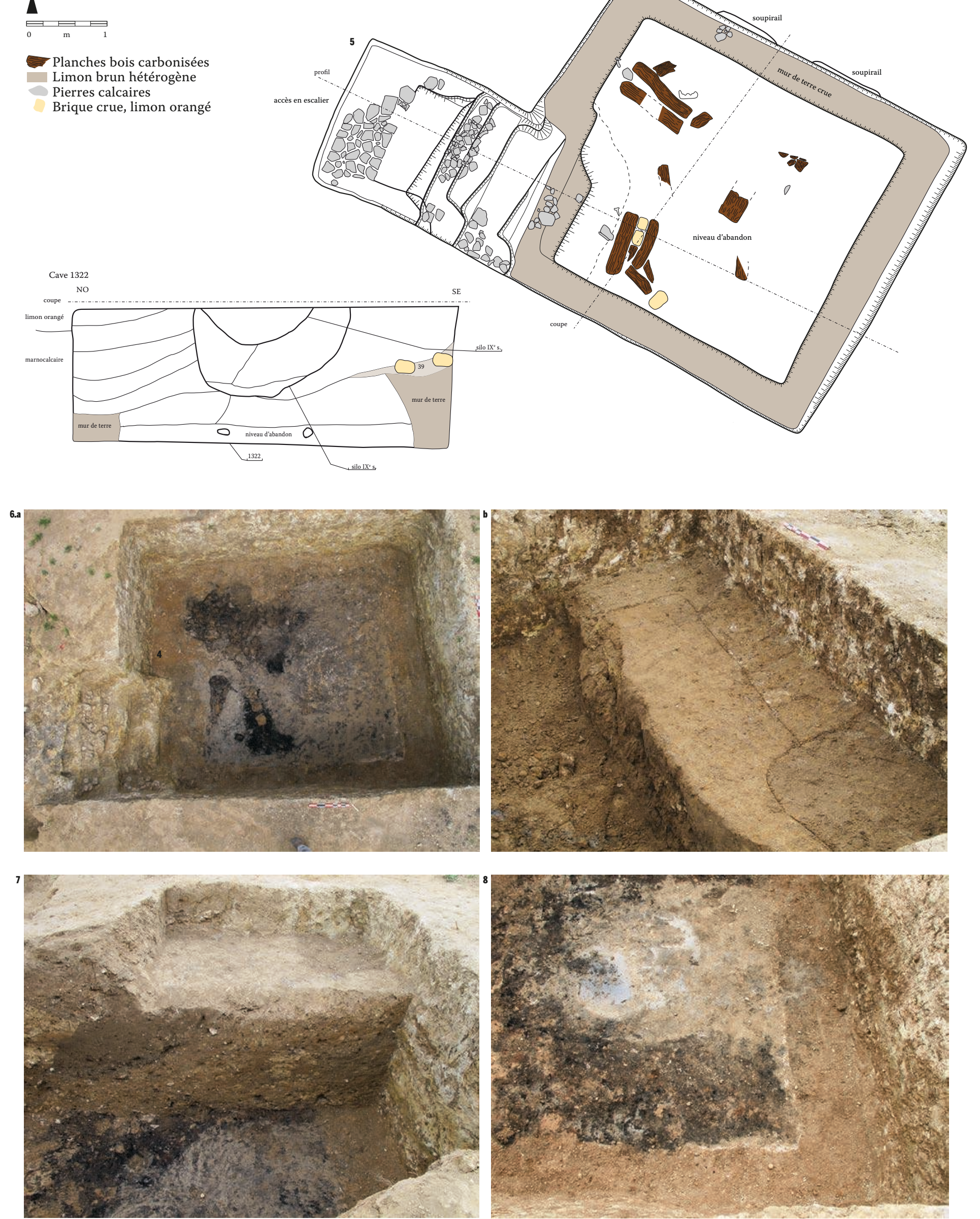


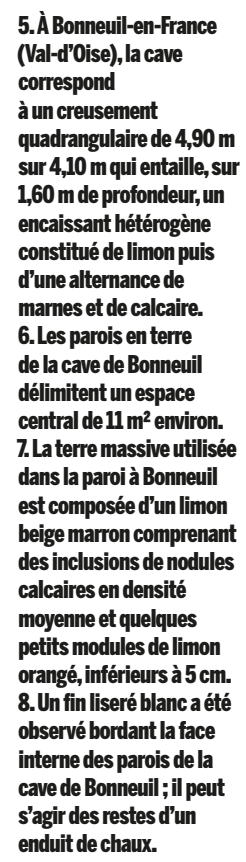

conservés laissent envisager que la forme des briques était carrée et plutôt régulière. L'utilisation d'un gabarit pour les façonner est probable.

D'autres fragments de brique ont été observés dans les niveaux d'effondrement. Ils sont plus petits et de nature différente. Deux textures se distinguent, l'une correspond à du limon orangé, sans dégraissant visible, équivalent au limon encaissant, et l'autre est plus beige brun légèrement hétérogène. Le module de ces derniers groupes de brique parait davantage cubique. Ces éléments peuvent constituer les restes d'un aménagement supérieur de la cloison ou d'une portion en élévation. Les tamisages du niveau d'abandon ont révélé un grand nombre de graines minéralisées pouvant provenir de fumiers utilisés comme dégraissant dans la mise en ouvre des briques. La différence de forme et de nature entre les briques plates d'argile verte et les cubiques limoneuses s'explique peut-être par l'usage du cellier et par une fonction particulière conférée à cette cloison. Bien que l'une des loges dessinée par la cloison ait livré un sédiment plus charbonneux que l'autre, ni l'analyse carpologique, ni le mobilier céramique n'ont permis d'identifier une utilisation particulière. Aucun indice ne nous permet d'avancer d'hypothèse quant aux activités menées dans ce cellier.

\section{Des parois en terre massive à Bonneuil}

L'occupation gallo-romaine de Bonneuil-enFrance, à « la Fontaine Plamont », n'est pour l'heure pas caractérisée car la fouille a concerné moins d'un quart du site ${ }^{\mathbf{3}}$ (Bruley-Chabot, 2015). Les structures de cette période se concentrent principalement à la rupture d'un versant de la vallée du Croult ; leur état de conservation témoigne d'une faible érosion. Outre la cave, le site compte aussi une structure de séchage de grains dont le creusement en sape nous est parvenu intégralement.

L'accès à l'intérieur de la cave s'effectue au moyen d'un escalier de cinq marches creusées dans l'encaissant, parementé de quelques pierres de calcaire sur les côtés et les bordures. Deux soupiraux complètent l'inventaire des aménagements creusés. Ces deux entailles rectangulaires, au profil évasé, sont localisées sur le haut de la paroi orientale. Les aménagements internes se singularisent par l'usage combiné de deux types de terre à bâtir : de la terre massive et des briques [ill. 5].

La terre massive a été observée en position primaire sur tout le pourtour intérieur de la cavité. Cette mise en œuvre correspond à une bande de $60 \mathrm{~cm}$ de large et reconnue sur un peu plus d'un mètre de hauteur [ill. 6]. Au-devant de l'escalier, la couche, visible sur une faible épaisseur, fait office de palier. Hors zones d'effondrement, les limites sont rectilignes et forment des parois verticales et lisses. La composition du mélange de terre est homogène sur la hauteur et en plan, quels que soient les côtés [ill. 7]. L'emploi de végétaux n’a pas sous la direction de Gaëll Bruley-Chabot. été constaté à l'œil, il ne peut cependant pas être exclu. Le long des parois et aux angles, il n'a été observé aucune limite ni joint, que ce soit lors des nettoyages en plan ou en coupe. Ces critères, absence de joint et homogénéité du mélange, privilégient l'hypothèse de l'emploi de terre massive. Nous ne pouvons déterminer le mode de mise en œuvre, à savoir coffrage ou façonnage [ill. 8]. La constitution de ce parement de terre a permis vraisemblablement de stabiliser les bordures de l'excavation, instables compte tenu de l'hétérogénéité de l'encaissant marno-calcaire. D'autres raisons peuvent être envisagées, comme la recherche des conditions hydrométriques ou de température particulières etc., mais nous n'avons aucun indice fonctionnel pour évaluer les objectifs poursuivis par les constructeurs. Le mobilier trouvé dans la première couche d'abandon (céramique, clous de menuiserie et de charpente) ne caractérise pas l'utilisation de la structure.

Les couches de comblement de l'excavation montrent plusieurs phases de destruction et d'effondrement des constructions en terre. Ces observations permettent de penser que les parois devaient être plus hautes que l'élévation conservée. Elles couvraient sans doute une grande partie de la hauteur de la cave.

Des fragments d'éléments modulaires, également en terre crue, ont aussi été observés dans le premier niveau d'abandon [ill. 9]. Ces briques, trouvées en position secondaire dans le comblement, n'ont pas été utilisées pour des partitions à l'intérieur de la partie excavée du cellier. En revanche, elles ont pu compléter l'élévation de terre massive ou être employées dans l'élaboration d'aménagements internes de la partie supérieure [ill. 10].

\section{Une confusion des genres}

Outre l'érosion, la reconnaissance des constructions en terre pâtit des confusions possibles avec des indices liés aux phases de fondation ou de récupération et matérialisés par des tranchées. Dans les deux exemples détaillés plus haut, la première interprétation qui a été posée, lors de l'apparition des couches de terre, a été celle d'une tranchée de récupération d'un mur en pierre. Les murs de pierre sont plus couramment rencontrés et ils font parfois l'objet de récupération de leurs blocs. Les tranchées creusées pour leur mise en place sont rectilignes comme les couches observées à Épiais et Bonneuil. Le fond des tranchées de fondation peut être nivelé au moyen d'un apport de terre. Ici, les hauteurs conservées sont trop importantes pour qu'il s'agisse d'une couche de réglage. Les tranchées de récupération ne sont quant à elles pas toujours aussi régulières. Et la collecte de matériau ne se cantonne parfois qu'à une portion de la construction, la partie sommitale (pour éviter de creuser trop profondément) ou une partie localisée du mur (en fonction du besoin). Lanalyse stratigraphique 


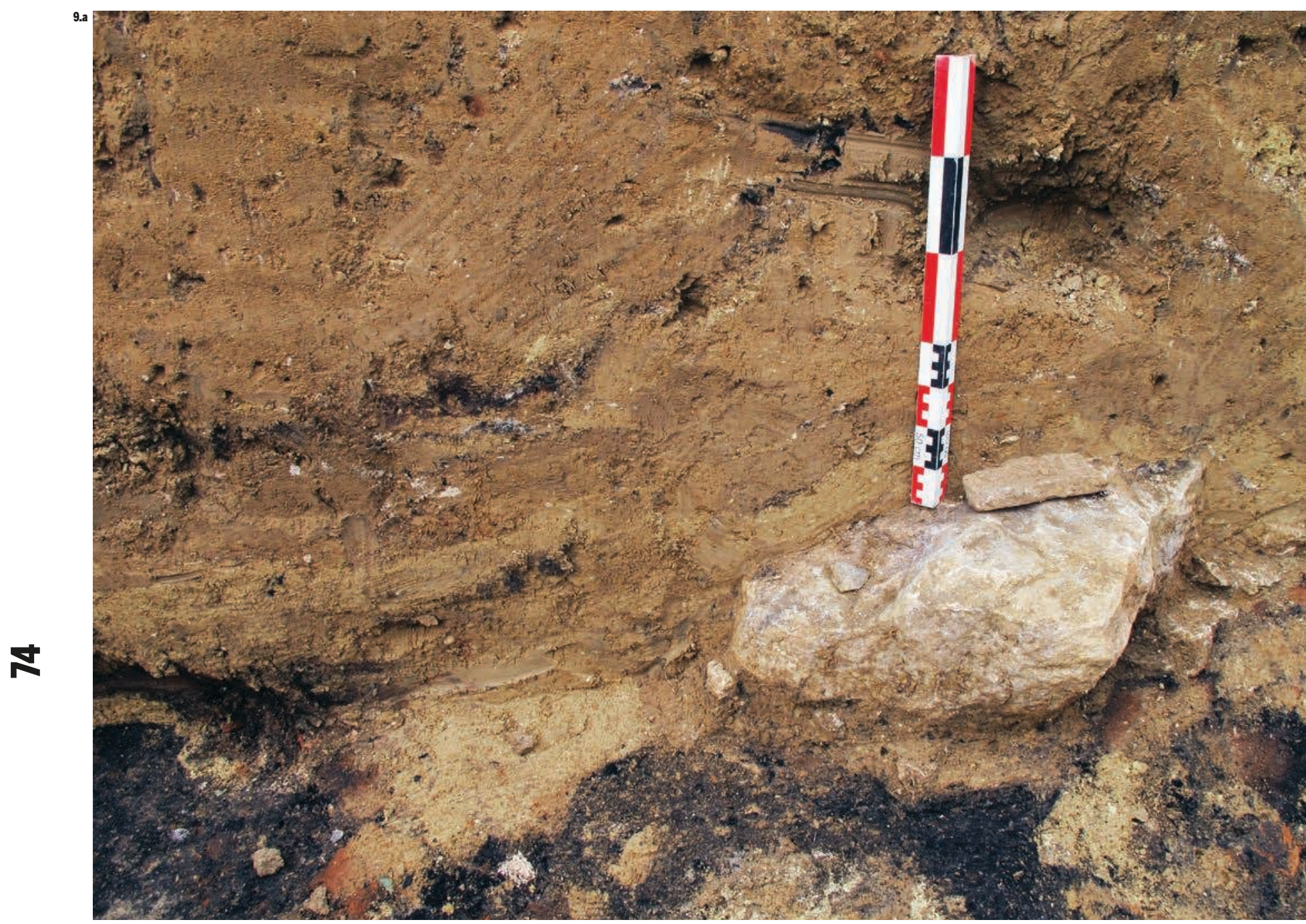

9. Les briques crues

quadrangulaires observées

dans la couche d'abandon

de la cave de Bonneuil sont

constituées d'un limon

jaune carbonaté, sans

mélange ni dégraissant

apparent (une lame mince

a été réalisée pour vérifier

les caractéristiques d'un

éventuel malaxage).
10. À Bonneuil, la présence

de briques crues dans la

couche d'abandon laisse

penser que la cave pouvait

être surmontée d'une

supertructure qui lui était

propre ou s'inscrire dans

un bâtiment plus vaste

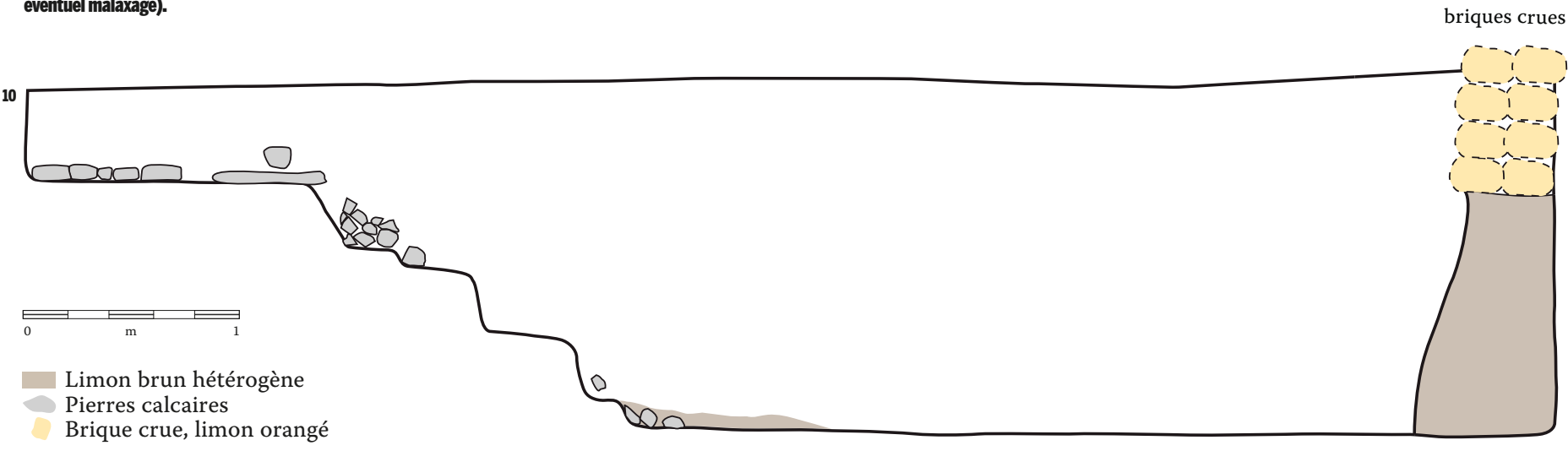

mur de terre crue en place 


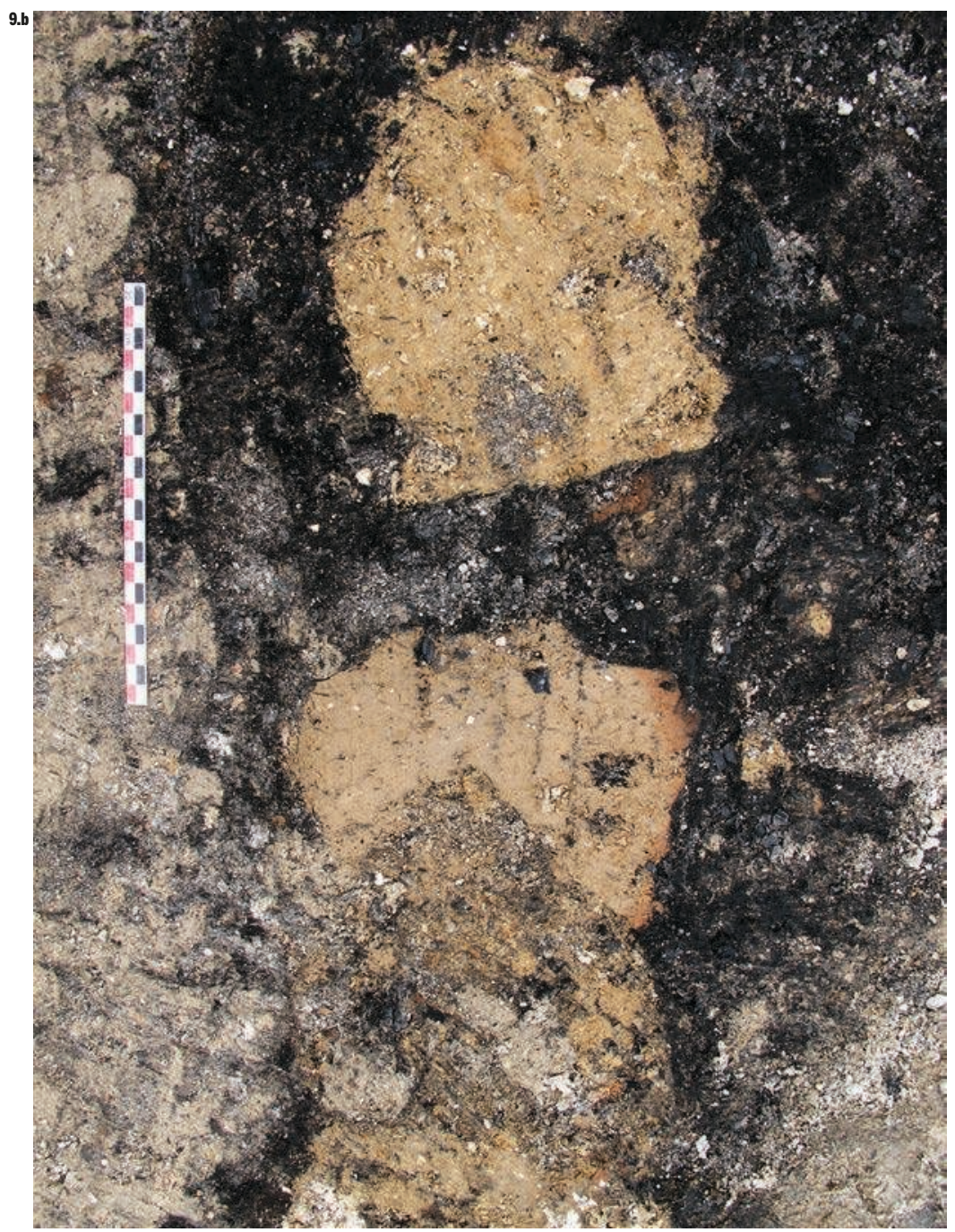

est donc déterminante. Elle doit distinguer ce qui est « creusement » de ce qui est « construit ». Énoncé de la sorte, cela paraît simple. Mais l'analyse peut être complexe lorsque toutes les couches correspondent à des sédiments très comparables. La confrontation des observations en coupe et en plan est indispensable et c'est souvent après démontage que la construction en terre devient évidente.

Les confusions d'interprétation se rencontrent aussi lors de l'analyse des restes comme dans le cas de la présence de graines minéralisées trouvées dans la couche d'abandon du cellier d'Épiais. Dans une optique liée à la reconnaissance des activités agricoles, on peut envisager de comprendre cette couche comme le témoignage d'une phase de relégation du cellier en fosse à fumier, tandis qu'en suivant une interprétation liée à une construction de terre effondrée, les mêmes graines minéralisées peuvent être considérées comme provenant du fumier employé comme dégraissant.

\section{L'extraction et le choix du matériau}

Dans les deux cas étudiés, l'extraction sur place a été privilégiée. Les recouvrements observés sur ces deux sites sont principalement constitués de
7. Lestimation utilise la cylindre, avec $12 \mathrm{~m}$ de diamètre et $70 \mathrm{~cm}$ de hauteur. limon orangé dont l'épaisseur dépasse rarement $2 \mathrm{~m}$. Ce limon surmonte le calcaire et les marnes. Des matériaux très différents peuvent donc être collectés sur place. À Bonneuil, la position sur le versant permet même un accès aux différents matériaux sans avoir à creuser profondément, il suffit de se déplacer sur la pente pour obtenir là du limon, là de la marne ou du sable. À Épiais, comme il s'agit du plateau, il faut creuser en profondeur pour se fournir en limon plus ou moins argileux puis en calcaire et marnes.

Les briques carrées du cellier d'Épiais, qui sont argileuses et de teinte gris vert, n'ont rien à voir avec le limon orangé rencontré sous la terre végétale. La couche sous-jacente est un limon plus gris qui reste néanmoins sableux ${ }^{4}$. Ce niveau comprend peut-être des zones plus argileuses. Par contre, des compositions, équivalentes à celle des briques, ont été observées dans le comblement de plusieurs grandes fosses comme dans le puits voisin ( $n^{\circ} 4167$ ) ou dans une vaste fosse fouillée à Mauregard, à $280 \mathrm{~m}$ de là ${ }^{\mathbf{5}}$ [ill. 11]. La nature argileuse des briques est-elle naturelle ou a-t-elle été acquise par décantation ? Le volume nécessaire à la confection de la cloison d'Épiais est estimé à $0,4.4 \mathrm{~m}^{3}$.

La couleur marron gris de la terre à bâtir de la cave de Bonneuil peut provenir du mélange, de l'opération de malaxage, mais aussi de l'extraction d'un matériau déjà « altéré » comme celui d'une fosse en cours de comblement. Il n'est pas rare que de grandes fosses d'extraction soient recreusées au fur et à mesure de leur comblement (par effondrement ou colluvionnement). Une fosse (1055) située à $40 \mathrm{~m}$ du cellier a pu servir de lieu d'extraction voire de zone de malaxage. Il s'agit d'une vaste fosse en cuvette, de $12 \mathrm{~m}$ de diamètre environ et $70 \mathrm{~cm}$ de profondeur. Ses contours sont irréguliers car elle a été creusée en plusieurs étapes. Les couches observées dans son comblement sont hétérogènes. Ouverte dès la période gallo-romaine, la fosse cesse d'être utilisée au $\mathrm{VI}^{\mathrm{e}}-\mathrm{VII}^{\mathrm{e}}$ siècle avec l'installation de fours culinaires en bordure. La quantité de terre nécessaire aux parois de la cave peut être estimée à $10 \mathrm{~m}^{3}$ environ ${ }^{6}$. La fosse a quant à elle permis d'extraire un minimum de $13 \mathrm{~m}^{3}$ de matériau?

Comme cela a été signalé précédemment, la conservation en position primaire a été possible à Épiais et Bonneuil parce que les aménagements en terre ont été construits dans des structures excavées. Les sites stratifiés sont les plus à même de livrer des exemples d'élévations construites en terre et conservées : celui de l'Institut Curie (Paris $5^{\mathrm{e}}$ arr., Busson et al., 2013, p. 100-101) recelait par exemple un mur de terre sur un soubassement constitué d'un blocage de pierre allié à un parement de briques crues [ill. 12]. En revanche, dans le cas d'occupations sans élévation conservée, un bâtiment à murs de terre ne laisse généralement de traces au sol que s'il possède des soubassements (sablières,
م્ّ 


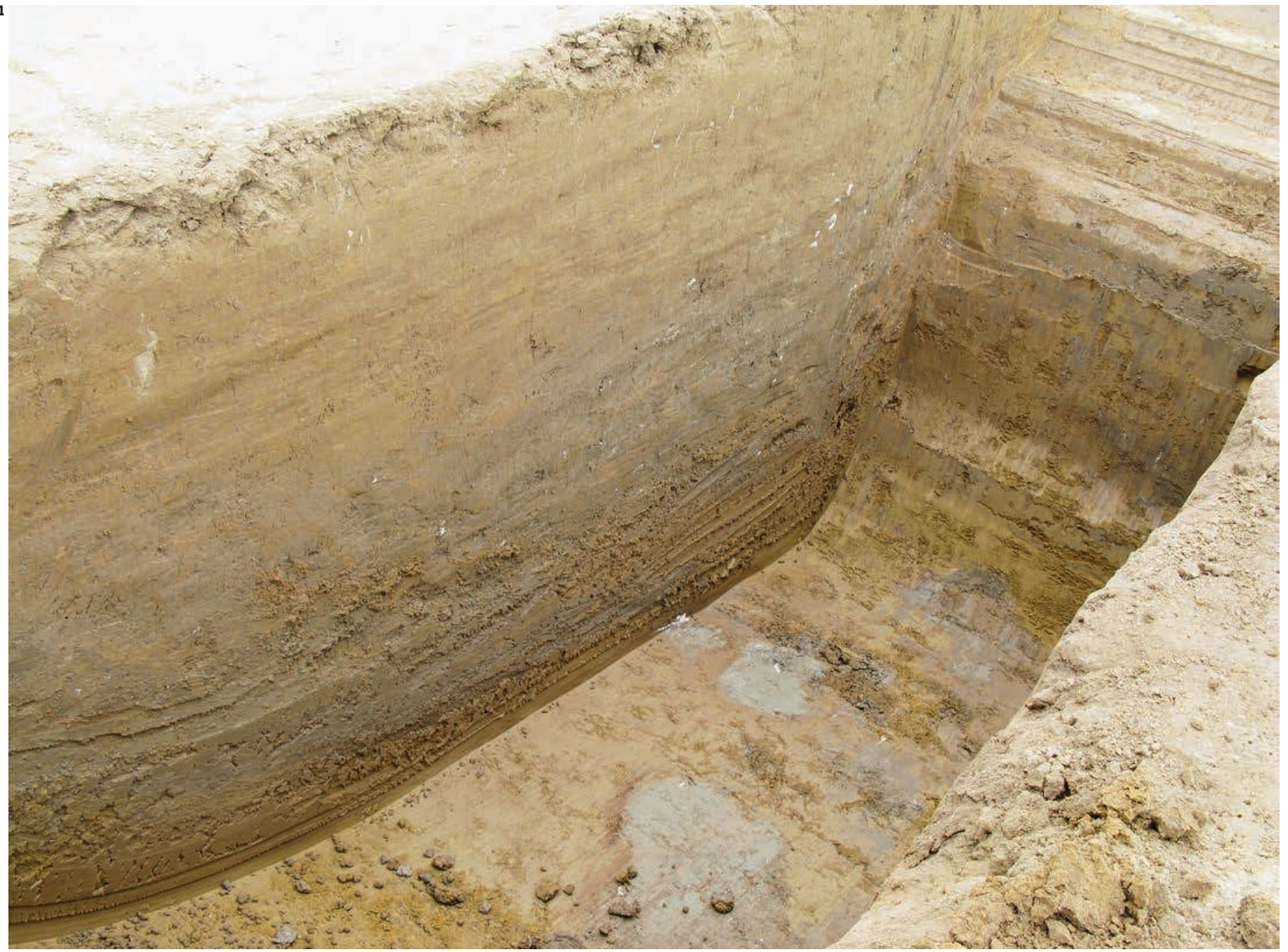

11. La fouille du site des Moulins à Mauregard (Seine-et-Marne) a mis au jour une fosse

d'extraction d'une argile dont les caractéristiques se rapprochent de celle utilisée à Épiais.
8. Par exemple : Roissyen-France « la Croix de Montmorency », SaintPathus « les Petits Ormes », Gonesse «ZAC des Tulipes», Longjumeau

« le Champtier des

Cerisiers "... (rapports consultables sur http:// dolia.inrap.fr/). solins de pierre ou tranchées de fondation). Mais la réciproque ne fonctionne pas systématiquement, la présence de ceux-ci ne permet pas d'émettre l'hypothèse d'une construction de terre. L'une des pistes pour conforter cette supposition réside peut-être dans l'estimation des besoins en terre que requièrent ces bâtiments. Partant du postulat qu'un mur de terre crue (en brique ou terre massive) nécessite davantage de sédiment qu'une paroi de torchis sur clayonnage, est-il possible de lier la présence de construction en terre à la mise au jour de vastes fosses d'extraction? Cette quantification du volume de terre extraite est à confronter évidemment au nombre de bâtiments sur poteaux repérés.

Ainsi pourrait s'expliquer la présence des grandes fosses d'extraction observables sur bon nombre de sites gallo-romains en Île-de-France ${ }^{\mathbf{8}}$. Le réexamen des cas permettrait peut-être d'identifier des choix architecturaux fonctionnels ou liés à une période chronologique plus qu'à une autre. Si les solins constitués de moellons se rencontrent durant toutes les phases de la période romaine, les vastes excavations, quant à elles, se multiplient à partir $\mathrm{du}$ III $^{\mathrm{e}}$ siècle et laissent envisager l'existence de constructions en terre massive (ou briques) plus répandues qu'on ne l'imagine. 

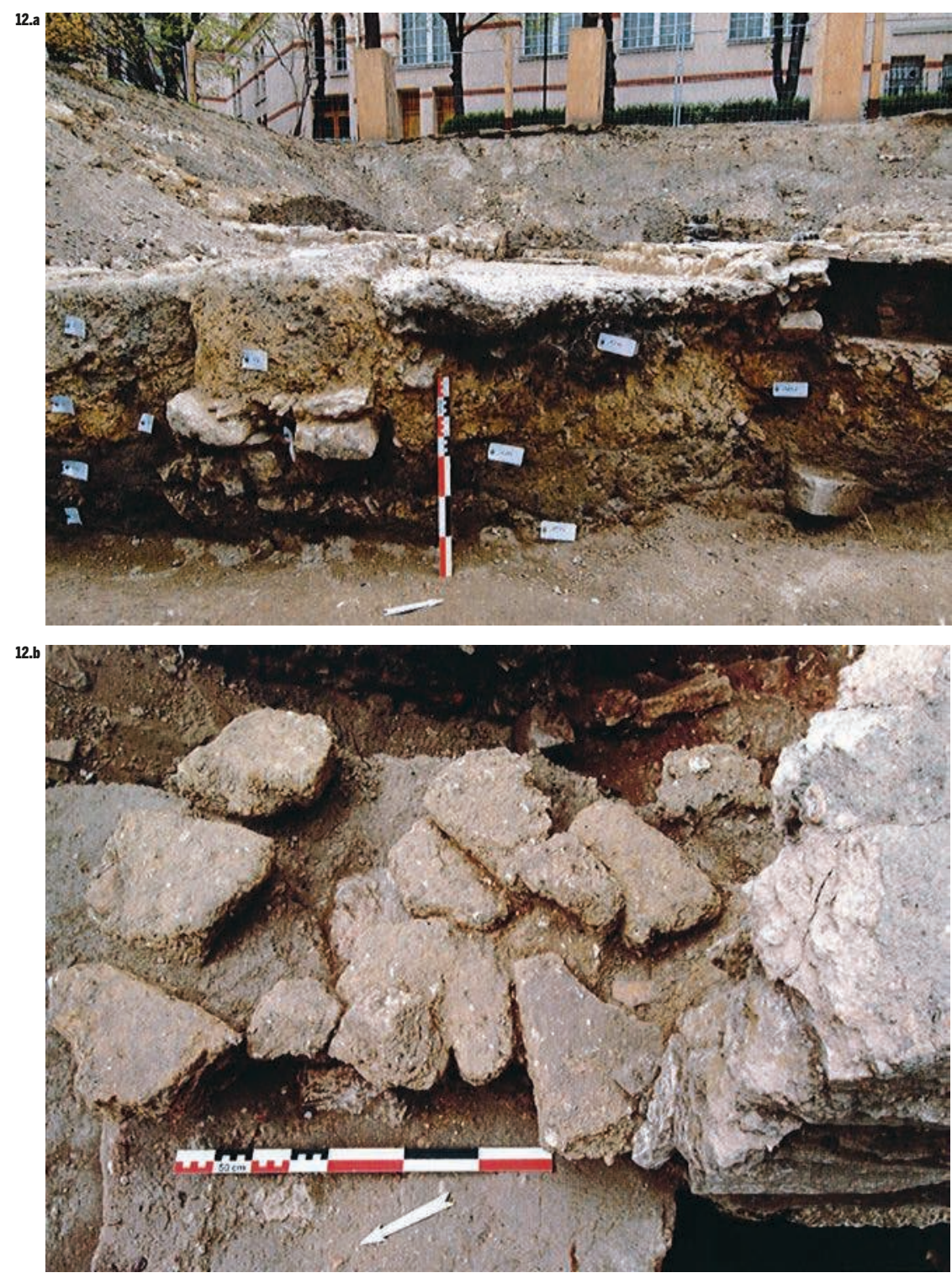

12. Mur d'hypocauste daté du II siècle (a)

composé en partie de

briques crues (b) sur

le site de l'Institut Curie,

à Paris.
Références bibliographiques

AURENCHE O. ET COLL., 2011, «Essai de classification des modalités de mise en œuvre de la terre crue en parois verticales et de leur nomenclature », in Chazelles C.-A. De, Klein A. et Pousthomis N. (DIR.), Les cultures constructives de la brique crue. Échanges transdisciplinaires sur les constructions en terre crue, 3, Actes du colloque international de Toulouse, 16-17 mai 2008, Montpellier, éditions de l'Espérou, p. 13-34.

Bruley-ChABOt G., 2013, Épiais-lès-Louvres (Vald'Oise), « la Fosse » : A 104, contournement est de l'aéroport de Roissy, rapport d'opération, t. 5, Une occupation gallo-romaine, II ${ }^{e}$-début IV siècle, atelier métallurgique - relais routier? Inrap-SRA Île-deFrance, $402 \mathrm{p}$.

Bruley-Chabot G., 2015, Bonneuil-en-France (Vald'Oise), « Aéroport du Bourget/Fontaine

Plamond » phase 1, rapport d'opération, Inrap-SRA Île-de-France, 2 vol. (PAO en cours).

Busson D. et al., 2013, Paris 5e, Institut Curie:

Laboratoire de biologie du développement: 22 rue d'Ulm, du 9 au 11 rue Pierre et Marie Curie, 193 rue Saint-Jacques, rapport d'opération, Inrap-SRA Île-de-France, 2 vol. $(165,367$ p.)

DUVERNAY T., 2003, «La construction en terre crue: potentiel des restes en position secondaire. Le cas d'un site rural du Bassin Parisien. Varennes-surSeine et Ville-Saint-Jacques (Seine-et-Marne)», in Chazelles C.-A. De, Klein A. (Dir.), Terre modelée, découpée ou coffrée. Matériaux et modes de mise en ouvre. Échanges transdisciplinaires sur les constructions en terre crue, 1 , Actes de la table-ronde de Montpellier 17-18 novembre 2001, Montpellier, éditions de l'Espérou, p. 55-71. 